\title{
Trans-Thoracic Echocardiography during VA-ECMO: May the Supra-Sternal Notch View Detect a Too-Proximally Watershed Area?
}

\author{
Enrico Giustiniano, $M D^{1^{*}}$ and Maurizio Cecconi, MD, FRCA, FFICM, MD(Res) $)^{1,2}$ \\ ${ }^{1}$ Department of Anesthesia and Intensive Care Units, Humanitas Clinical and Research Center-IRCCS, Italy \\ ${ }^{2}$ Department of Biomedical Science, Humanitas University, Italy
}

*Corresponding author: Enrico Giustiniano, Department of Anesthesia and Intensive Care Units, Humanitas Clinical and Research Center, IRCCS, via Manzoni 56-20089, Rozzano, Milan, Italy, Tel: +39-02-8224-7459, Fax: +39-02-8224-4190

\section{Keywords}

Echocardiography, VA-ECMO, Watershed area

\section{Brief Report}

Veno-Arterial Extra-Corporeal Membrane Oxygenation (VA-ECMO) in adults has grown over the last decades as a life-saving procedure. Trans-thoracic (TTE) and trans-esophageal (TEE) echocardiography are recommended tool to manage such cases [1,2].

In VA-ECMO the suction cannula is generally inserted into a femoral vein and it is advanced just beyond the caval-atrial junction (Figure 1). The return cannula is inserted into a femoral artery and advanced into the distal portion abdominal aorta [2]. According the residual left ventricle (LV) function, the two blood flows (one from the LV and one from the return cannula) will mix. The mixing point of the two flows may be closer to the aortic valve if the LV contractility is severely failing or more distal when LV function is improving and/ or the ECMO-pump rate is reduced. The proximal and the distal blood flows are differently oxygenated. When the mixing point (i.e. the watershed area) is too proximal (along the aortic arch) the Harlequin Syndrome (HS) occurs. HS consists of two differently oxygenated portions of the body: the upper portion hypoxic due to the poorly oxygenated blood arriving from the LV and the lower well oxygenated one perfused by the blood coming from the ECMO oxygenator and pumped into the aorta. The cerebral and myocardial circulations will receive less oxygenated blood, exposing the patient to the risk severe complications [3].

A 35-years-old female patient suffering from myocarditis after bone marrow transplant for lymphoblastic leukemia underwent V-A ECMO due to a severe Left Ventricle disfunction which developed abruptly. Given the rapid clinical worsening we opted for the extracorporeal circulation support as first choice.

Despite TEE has been reported as a minimally complicated exam in case of thrombocytopenia [4], we checked the heart function by TTE (Philips Sparq Core 77, Philips Ultrasound, Bothell, WA, USA) because of a very low platelets count and a very good trans-thoracic Ultra-Sounds (US) window. We found that from the supra-sternal notch (SSN) view [5], a spontaneous contrast image referable to the Watershed Area was detectable at the level of the ascending portion of aortic arch proximally to the origin of the right subclavian artery (Figure 1 and Video 1). The image had as a proximal-concavity plume shape we hypothesized caused by the "smoke effect" that the two mixing flows created. At the same time the oxygenation of the blood from the right radial artery line was quite lower than the blood from the pump.

Two causes can make the water-shed point move more distally: A) The Left Ventricle performance im-

Citation: Giustiniano E, Cecconi M (2020) Trans-Thoracic Echocardiography during VA-ECMO: May the Supra-Sternal Notch View Detect a Too-Proximally Watershed Area?. Int J Anesthetic Anesthesiol 7:113. doi.org/10.23937/2377-4630/1410113

Accepted: November 17, 2020: Published: November 19, 2020

Copyright: (C) 2020 Giustiniano E, et al. This is an open-access article distributed under the terms of the Creative Commons Attribution License, which permits unrestricted use, distribution, and reproduction in any medium, provided the original author and source are credited. 


\section{A- Supra-Sternal Notch view}

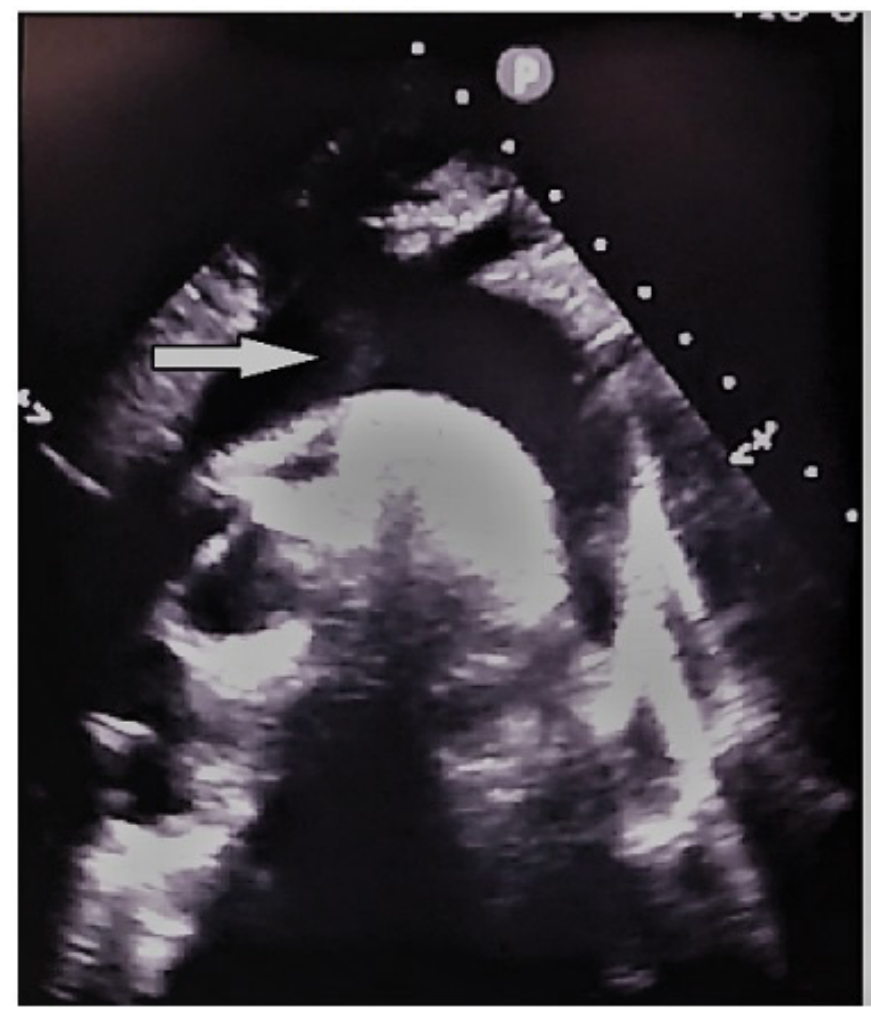

B - Sub-costal view: he tip of the venous cannula

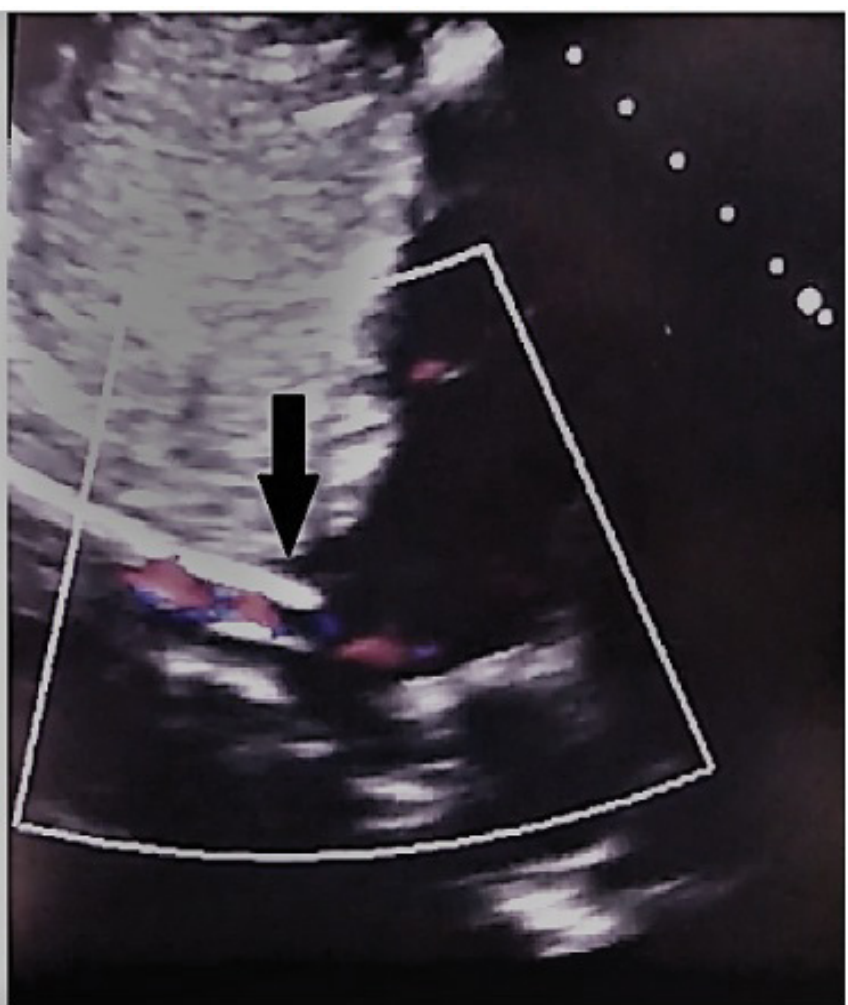

Figure 1: The supra-sternal notch and sub-costal trans-thoracic echocardiographic views.

White arrow: The point of the aortic arch where native blood flow and the return cannula blood flow converge.

Black arrow: The aspiration cannula just beyond the inferior vena cava - right atrium junction.

proving (also due to inotrope drug and/or Intra-Aortic Balloon Pump - IABP - insertion); B) Reducing the ECMO return flow by acting on the pump rate. Furthermore, the HS may recover when: 1 ) The oxygenation of pulmonary venous blood increases by adjusting the ventilator settings or performing lung recruitment maneuver, provided that the right ventricle function has improved;

2) Changing VA-ECMO to VV-ECMO (venous-venous $\mathrm{ECMO}$ ) if cardiac function restored.

In our case VV-ECMO was not practicable because we have not sufficient experience with it.

Despite Dobutamine administration LV performance required the insertion of IABP (1:1 rate) too. Moreover, the cardiac-surgeon consultant did not considered to unload the LV by an Impella ${ }^{\circledR}$, basing on his own experience and because IABP insertion would be more appropriate to increase the LV function. After IAPB insertion, at a second-look by TTE-SSN we didn't find the plume-shed image we found at the first US-exam, but unfortunately we did not save the image. Anyway we considered it as a sign of improved LV function. Moreover, aiming at further improving LV performance to wean the patient from V-A ECMO as soon as possible, we swapped from Dobutamine to Levosimendan, successfully. Then ECMO pump support was progressively reduced and patient weaned from the extracorporeal circulation in a week and discharged to ward after 10 days.
At our knowledge, the Watershed Area has never been described by trans-thoracic echocardiography. Buchtele, et al. described it by TEE [6].

In our case it was an incidental finding during the TTE exam. The greatest limitation to our hypothesis is the lack of a comparable imaging after LV performance increased. Anyway, if the SSN will be confirmed to be eligible to detect a too-proximally Watershed Area, it would be strategically very helpful to manage such severe clinical situation non-invasively, also from a prognostic assessment point-of-view.

\section{Source of Funding}

None.

\section{Disclosure}

Declarations of interest: None.

\section{References}

1. Combes A, Brodie D, Bartlett R, Brochard L, Brower R, et al. (2014) Position paper for the organization of extracorporeal membrane oxygenation programs for acute respiratory failure in adult patients. Am J Respir Crit Care Med 190: 488-496.

2. Douflé G, Roscoe A, Billia F, Fan E (2015) Echocardiography for adult patients supported with extracorporeal membrane oxygenation. Crit Care 19: 326.

3. Sangalli F, Patroniti N, Pesenti A (2014) ECMO-extracorporeal life support in adults. Springer-Verlag Italia. 
4. Kitkungvan D, Kalluru D, Lunagariya A, Sanchez L, Yusuf SW, et al. (2019) Safety of transesophageal echocardiography in patients with thrombocytopenia. J Am Soc Echocardiogr 32: 1010-1015.

5. Mitchell C, Rahko PS, Blauwet LA, Canaday B, Finstuen JA, et al. (2019) Guidelines for performing a comprehensive transthoracic echocardiographic examination in adults:
Recommendations from the american society of echocardiography. J Am Soc Echocardiogr 32: 1-64.

6. Buchtele N, Staudinger T, Schwameis M, Schörgenhofer C, Herkner H, et al. (2020) Feasibility and safety of watershed detection by contrast-enhanced ultrasound in patients receiving peripheral venoarterial extracorporeal membrane oxygenation: A prospective observational study. Crit Care 24: 126. 